\title{
Coexistent squamous cell carcinoma and tuberculosis in the cervix: a rare case report
}

\author{
Mousumi Sharma ${ }^{1}$, Siraj Ahmed ${ }^{1}$, Amal Chandra Kataki ${ }^{2}$, Anupam Sarma ${ }^{1}$ \\ ${ }^{1}$ Department of Pathology, Dr. B. Borooah Cancer Institute, Guwahati, India. \\ ${ }^{2}$ Department of Gynaecologic Oncology, Dr. B. Borooah Cancer Institute, Guwahati, India.
}

Received June 04, 2014; Revised July 04, 2014; Accepted July 05, 2014; Published Online July 10, 2014

\section{Case Report}

\begin{abstract}
Tuberculosis may coexist with malignancy. But coexistence of tuberculosis and cancer in cervix is extremely rare. We present a rare case of coexistent squamous cell carcinona and tuberculosis of uterine cervix in a 35 years old lady who presented with chief complaints of white discharge and bleeding per vagina since 1 year. Histopathological findings of carcinomatous nests along with epithelioid granuloma, langhans type of giant cells and positive acid fast bacilli in the tissue section give authenticity to our diagnosis. Tuberculosis complicating malignancy may occur in regions with high prevalence of this disease. Hence, proper diagnosis of tuberculosis in a patient with cancer assumes importance as it may add to the surveillance of the patient.
\end{abstract}

Keywords: Tuberculosis; Malignancy; Cervix

\section{Introduction}

Tuberculosis and malignancy, both represent global threat claiming millions of lives and inflicting formidable sufferings worldwide. ${ }^{1}$ In 2005, WHO reported a prevalence of 20 million cases of tuberculosis worldwide, out of which 15 million reside in developing countries like India. ${ }^{2}$ In comparison to extra genital tuberculosis, tuberculosis of female genital system accounts for minority of cases, $90 \%$ affecting female of reproductive age group. The most commonly affected region are endometrium and fallopian tubes. Tuberculosis of cervix is a rare disease accounting 5 - $24 \%$ of genital tuberculosis and $0.1-0.65 \%$ of all tuberculosis cases..$^{2-3}$ On the other hand, cervical carcinoma is the fourth most common cancer in women worldwide and India accounting for more than one fifth of global cervical cancer burden. ${ }^{4}$

Many cases of cervical tuberculosis have been reported in literature across the world, however, coexistent case of cervical carcinoma with cervical tuberculosis is extremely rare. Last case was reported in $1985 .{ }^{5}$ We diagnosed a case of cervical carcinoma with coexistent cervical tuberculosis during

Corresponding author: Mousumi Sharma; Department of Pathology, Dr. B. Borooah Cancer Institute, Guwahati, India.

Cite this article as:

Sharma M, Ahmed S, Sharma J, Kataki AC, Sarma A. Coexistent squamous cell carcinoma and tuberculosis in the cervix: a rare case report. Int J Cancer Ther Oncol 2014; 2(3):020314.

DOI: $10.14319 /$ ijcto.0203.14 our routine histopathological reporting in a 35 year old lady, which seems to merit reporting because of extreme rarity and pathological authenticity.

\section{Case report}

A 35 years old lady (Para 3 living 3), housewife by occupation, presented with chief complaints of irregular bleeding and white discharge per vagina since 1 year. There was history of weight loss. There was no history of any malignancy in the past or in the family. Patient did not have any other significant medical or surgical illness in the past and no suggestive family history.

On general examination, she had pallor. Per speculum examination revealed a large fungating mass replacing the cervix and extending up to the anterior vaginal wall and bleeding on touch. On bimanual examination, same growth was felt.

All routine hematological and biochemical parameters were within normal limit except low hemoglobin and raised ESR. HIV1/2 was non-reactive, chest skiagram was within normal limit.

CT scan showed a cervical growth extending to upper vaginal wall, lower endometrium, and bilateral parametrial and pel- 
vic extension. Cervical biopsy was taken and sent for histopathological examination. Microscopic examination of H\&E stained section showed sheets and nests of neoplastic cells with morphology of squamous cell carcinoma (large cell non keratinizing type) mixed with extensive epithelioid cell granulomas with langhans' type of giant cells and focal caseous necrosis (Figure 1-3) suggesting assosciated tuberculosis. However for confirmation, two serial sections of biopsy fragment were stained with ZeihlNeelson (ZN) stain. On examination, straight to slightly curved beaded acid fast bacili were seen (Figure 4). These findings were sufficient to diagnose the case as squamous cell carcinoma (large cell non keratinizing type) of cervix with coexistent cervical tuberculosis.

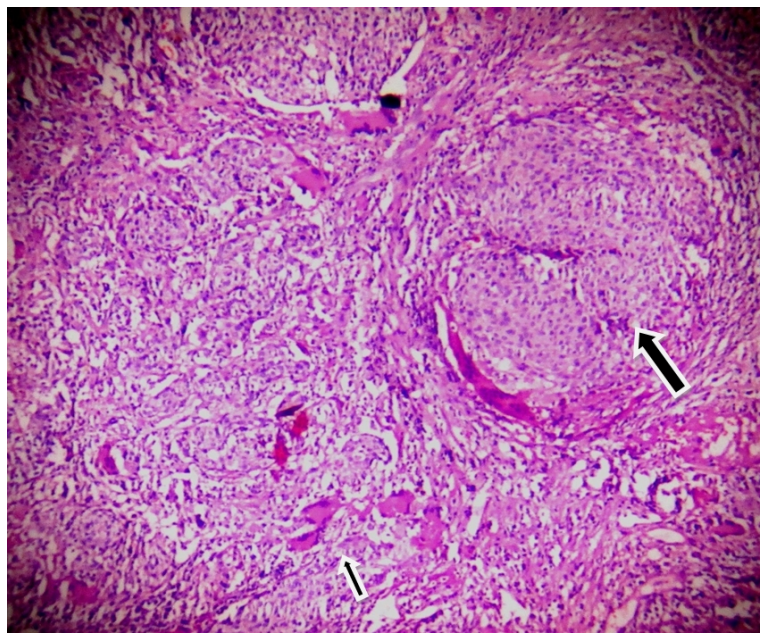

FIG. 1: Low power view showing tumor cell nests (thick arrow) along with many Langhans' type of giant cells (thin arrow).

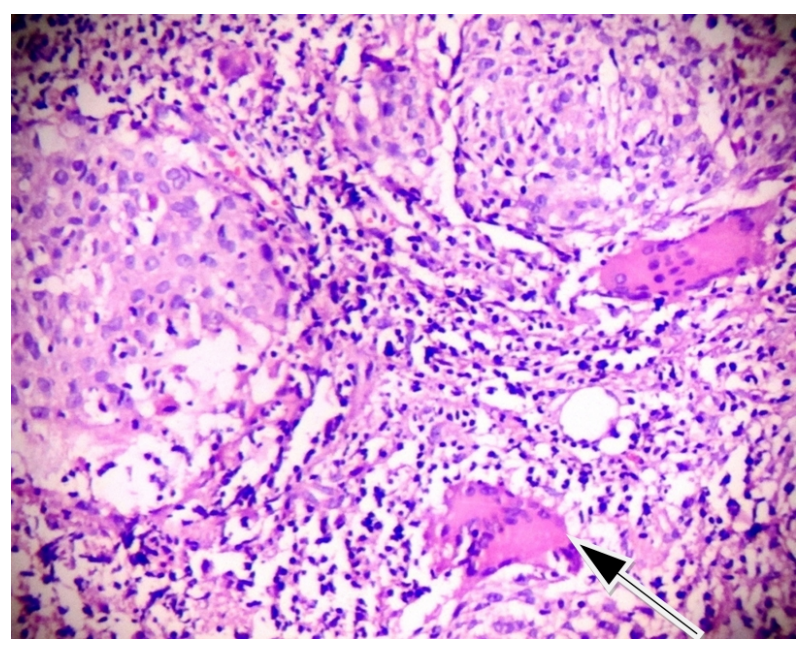

FIG. 2: High power view showing tumor cell nests with Langhans giant cells (arrow).

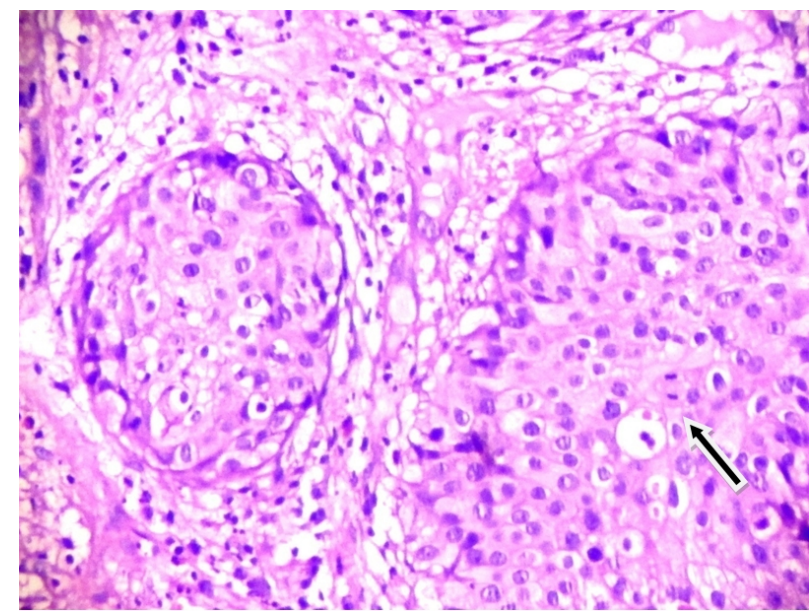

FIG. 3: High power view showing pleomorphic cancer cell nests with hyperchromatic nuclei and mitosis (arrow).

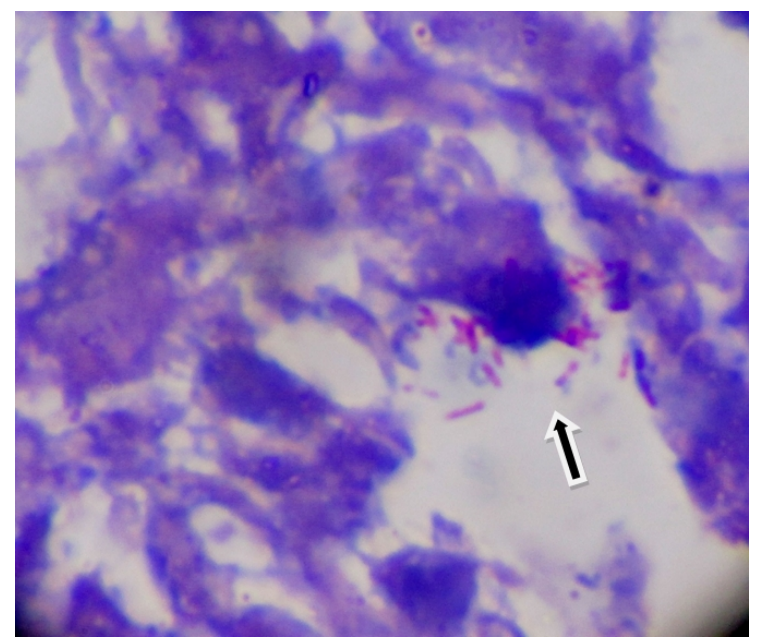

FIG. 4: ZN stain showing positive acid fast bacilli (oil immersion).

Patient was put on anti-tubercular treatment and planned for radiotherapy and we will follow up the case subsequently.

\section{Discussion}

Inspite of tuberculosis and cancer being global threat worldwide, coexistence of these two disease in the same patient and same site is rare. ${ }^{1}$ Few studies have been carried out and reported in literature. A review study revealed 47 case reports of coexistence of tuberculosis and malignancy in the same site, including 3 case reports of co-existent genital tuberculosis and malignancy (tubercular salpingitis and adenocarcinoma of fallopian tube; squamous cell carcinoma cervix and endometrial tuberculosis; and endometrial adenocarcinoma and genital tuberculosis). ${ }^{1}$ 
An oncology center in India has reported highest incidence of pulmonary tuberculosis and head and neck cancer (42\%) followed by GIT carcinoma (14.1\%), lung ca (13.8\%), hematological ca $(10.7 \%)$, reproductive ca $(0.3 \%)$ and miscellaneous (9\%). ${ }^{6}$ Rajaram et al. reported a case of co-existent squamous cell carcinoma cervix with endomerial tuberculosis. ${ }^{7}$ In another case report, association of co-existent extra-genital tuberculosis and gynecological tumor was reported. ${ }^{8}$

However, cervical tuberculosis is rare and co-existent carcinoma and tuberculosis of cervix is extremely rare. In 1985 , $\mathrm{Wu}$-Hsiung Chen last reported two cases of co-existent carcinoma and tuberculosis of cervix, after that no such case has been reported so far. ${ }^{5}$ So, we thought of reporting the present case because of extreme rarity and prognostic significance. On the basis of our experience with this case, it may be difficult sometimes to detect such coexistence in a busy routine histopathological reporting as occasional presence of giant cells in the vicinity of cancer and also other causes of granulomatous cervicitis may give a mistaken diagnosis. In our case, typical nests of cancerous cells and caseating granuloma with langhans' giant cells and positive acid fast bacilli give authenticity to our diagnosis.

Coexistence could be coincidence or reactvation of latent tuberculosis or fresh infection due to immunosupprresion. Rajaram et al. concluded that TB complicating a case of malignant disease may occur in regions with a high prevalence of disease; given the resurgence of tuberculosis worldwide, this association may not be that rare. ${ }^{7}$ Research on this association is going on, but the mechanism is yet to be elucidated. So special care is required during histopathological examination of carcinoma assosciated granulomatous cervicitis and one should go for simple AFB staining as this may significantly add to the surveillance of the patient.

\section{Conclusion}

The incidence of tuberculosis is increasing and due to this resurgence worldwide, coexistence of tuberculosis with malignancy may not be that rare. Clinically it may be difficult to detect due to overlapping symptoms, hence special care should be taken during histopathogical examination whenever there is high index of suspicion.

\section{Conflict of interest}

The authors declare that they have no conflicts of interest. The authors alone are responsible for the content and writing of the paper.

\section{References}

1. Falagas ME, Kouranos VD, Athanassa Z, Kopterides P. Tuberculosis and Malignancy. $Q \quad J M 2010$; 103:461-87.

2. Mehrangiz Hatami. Tuberculosis of the female genital tract in Iran. Arch Iranian Med 2005; 8:32-5.

3. Maj M Paprikar, Col M Biswas, Col S Bhattacharya, et al. Tuberculosis of cervix: Case report, MJAFI 2008; 64/3: 297-8.

4. Ferlay J, Soerjomataram I, Ervik M, Dikshit R, Eser S, Mathers C, Rebeloet al. (2013). Cancer incidence and Mortality Worldwide: IARC Cancer Base No.11 [Internet]. GLOBOCAN 2012 v 1.0, Lyon, France: International Agency for Research on Cancer, 2013. Available from http://globocan,iarc.fr

5. Hsu CT, Yang LC, Hsu ML, Chen WH, Lin YN. The coexistence of carcinoma and tuberculosis in the uterine cervix: report of 2 cases. Asia Oceania $J$ Obstet Gynaecol 1985; 11: 363-9.

6. Kumar RR, Shafiulla M, Sridhar H. Association of tuberculosis with malignancy at KIMIO -an oncology centre. Indian J Pathol Microbiol 1999; 42: 339-43.

7. Rajaram S, Dev G, Panikar N,et al. Postmenopausal bleeding: squamous cell carcinoma of cervix with coexisting endometrial tuberculosis. Arch Gynecol Obstet 2004; 269; 221-3.

8. Shamsunder S, Banerjee A, Mala YM, et al. Co-existent extrapulmonary, extragenital tuberculosis and gynaecological tumors: a reminders to pathologists. Int J Reprod Contracept Obstet Gynecol 2013; 2: 733-4. 